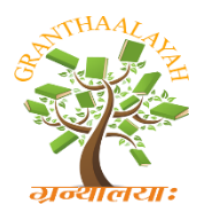

$$
\begin{gathered}
\text { INTERNATIONAL JOURNAL OF RESEARCH } \\
\text { GRANTHAALAYAH } \\
\text { A knowledge Repository }
\end{gathered}
$$

Science

\title{
ACUPUNCTURE TREATMENT IN PATIENT WITH INFERTILITY
}

\author{
Prof Dr Jihe Zhu ${ }^{1}$, BSc Blagica Arsovska ${ }^{1,2}$, BSc Kristina Kozovska ${ }^{1,3}$ \\ ${ }^{1}$ Faculty of Medical Sciences, University Goce Delchev, Shtip, Republic of Macedonia \\ ${ }^{2}$ Institutes of Biology, Faculty of Natural Sciences and Mathematics, Skopje, Republic of \\ Macedonia \\ ${ }^{3}$ Medicine Faculties, St. Cyril and Methodius University of Skopje, Republic of Macedonia
}

\begin{abstract}
Acupuncture as part of the Traditional Chinese Medicine (TCM) is used very often as a treatment option for patients struggling with infertility. Acupuncture can help in regulating the hormonal imbalances, relieve the stress and increase the blood flow to the ovaries and uterus. The treated patient is 30 year old woman diagnosed with polycystic ovarian syndrome (PCOS) and infertility. The patient was trying to get pregnant for a year and a half. The menstrual cycles were irregular and the patient was taking the medication Diane for 4 years. The hormonal analysis showed high FSH levels and low Estrogen and Progesterone. The patient has done 6 treatments in a period of two months and after the 6 th treatment she got pregnant. Treatments were done in a clinic for TCM and acupuncture in Skopje, Macedonia by a doctor specialist in acupuncture. Treatments were with duration of 30-45 minutes, done once weekly on points located on the both sides of the body. The treated points are: Ex-CA1 (Zigongxue), Gv20 (Baihui), St25 (Tianshu), St36 (Zusanli), Ren12 (Zhongwan), Ren6 (Qihai), Ren2 (Qugo), Sp6 (Sanyinjiao), Li4 (Hegu), Lv3 (Taichong) and Ashi points located on front side of the neck. Acupuncture as a treatment for infertility is very effective and gives excellent results by improving the hormonal levels, the menstruation and the ovulation in women, thus giving a chance to the patients to become pregnant.
\end{abstract}

Keywords: Traditional Chinese Medicine; Acupuncture; Infertility; Treatment.

Cite This Article: Dr Jihe Zhu, Blagica Arsovska, and Kristina Kozovska. (2018). "ACUPUNCTURE TREATMENT IN PATIENT WITH INFERTILITY." International Journal of Research - Granthaalayah, 6(12), 30-33. 10.29121/granthaalayah.v6.i12.2018.1073.

\section{Introduction}

Acupuncture as part of the Traditional Chinese Medicine (TCM) is used very often as a treatment option for patients struggling with infertility. Acupuncture can help in regulating the hormonal imbalances, relieve the stress and increase the blood flow to the ovaries and uterus. [1] Chinese medicine treats a wide range of disorders including infertility due to polycystic ovarian syndrome (PCOS). [2] 
PCOS is a complex condition characterized by menstrual irregularities, elevated androgen levels and small cysts on one or both ovaries. Symptoms of PCOS are acne, obesity, excessive hair growth, amenorrhea and infertility. Women struggling with PCOS have higher rates of anxiety, depression, endometrial cancer, type-2 diabetes mellitus, sleep apnea, cardiovascular disease and dyslipidemia. The factors may be genetic or poor diet, inactivity, toxins and other. The primary defects of PCOS are in the hypothalamic-pituitary axis, insulin action and secretion and ovarian function. [3]

In TCM, PCOS is described as genetic disorder with main symptoms of irregular periods and infertility due to anovulation. There are three main patterns of diagnosis of PCOS:

1) Kidney deficiency - it is considered as root cause for PCOS. Absence of period and ovulation are Kidney pathologies

2) Spleen deficiency - The Spleen is an organ that is related with insulin resistance, weight gain, transformation and transportation of the fluids in the body and metabolism of nutrients from the food. If the Spleen function is improved then the blood sugar can be regulated, the fat from the weight gain and the excess fluid accumulation from ovarian cysts can be resolved.

3) Liver stagnation - it manifests as Blood stasis or excess heat in the meridians. Blood stasis in the meridians causes hirsutism symptom, which is present in $70 \%$ of the cases with PCOS and excess heat causes acne.

Therefore, the three main organs treated with acupuncture are Kidney, Spleen and Liver. The main focus of the treatment when trying to improve fertility is to induce ovulation. Acupuncture for PCOS can be used as a stand alone treatment or in combination with herbal therapy, moxibustion or electro-therapy. [4]

\section{Case Report}

The treated patient is 30 year old woman diagnosed with polycystic ovarian syndrome (PCOS) and infertility ten years ago. The patient was trying to get pregnant for a year and a half. The menstrual cycles were irregular and the patient was taking the medication Diane for 4 years. The hormonal analysis showed high FSH levels and low Estrogen and Progesterone levels. The results from the hormonal analysis are shown in Table 1. The results from the partner's semen test are showing normozoospermia (normal results of the semen).

The patient has done 6 treatments in a period of two months and after the $6^{\text {th }}$ treatment she got pregnant. Treatments were done in a clinic for TCM and acupuncture in Skopje, Macedonia by a doctor specialist in acupuncture. Treatments were done indoor, on a room temperature, with duration of 30-45 minutes, done once weekly on points located on front side of the body. In the treatment were used fine sterile disposable needles with dimensions $0.25 \times 25 \mathrm{~mm}$ manufactured by Wuijuiang City Medical \& Health Material Co., LTD. The treated points are: Ex-CA1 (Zigongxue), Gv20 (Baihui), St25 (Tianshu), St36 (Zusanli), Ren12 (Zhongwan), Ren6 (Qihai), Ren2 (Qugo), Sp6 (Sanyinjiao), Li4 (Hegu), Lv3 (Taichong) and Ashi points located on front side of the neck. 
Table 1: Results from hormonal analysis done before the treatment

\begin{tabular}{|l|l|l|}
\hline Test & Results & Normal range \\
\hline FSH & 10.2 & $4.5-11.0 \mathrm{mIU} / \mathrm{ml}$ \\
\hline LH & 6.5 & $1.7-13.3 \mathrm{mIU} / \mathrm{ml}$ \\
\hline Estrogen & $<25.0$ & $30-120 \mathrm{pg} / \mathrm{mL}$ \\
\hline Progesterone & 0.398 & $0.95-26.6 \mathrm{ng} / \mathrm{ml}$ \\
\hline TSH & 2.0 & $0.4-4.3 \mathrm{MIU} / \mathrm{L}$ \\
\hline FT4 & 12.9 & $10.6-21.0 \mathrm{pmol} / 1$ \\
\hline Fe & 11.9 & $7.1-26.0 \mathrm{mmol} / \mathrm{l}$ \\
\hline
\end{tabular}

On figure 1 is shown UV scan of the uterus done after the $6^{\text {th }}$ treatment showing embryo in early pregnancy. The patient is in 14th gestational week so far.

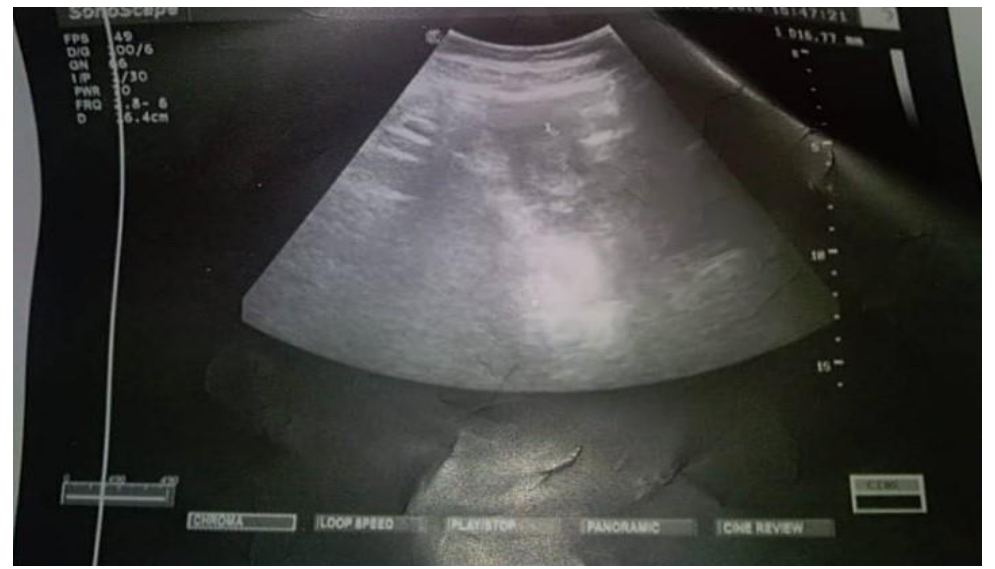

Figure 1: UV scan of uterus, showing embryo in early pregnancy

\section{Discussion}

A normal FSH level needed for a woman to conceive is typically below $10 \mathrm{mIU} / \mathrm{ml}$ In our practice we believe that for pregnancy to happen the FSH level needs to be between 4 and $8 \mathrm{mIU} / \mathrm{ml}$. The hormones are very complicated and intertwined and often one hormone is needed for another to be released. If one hormone is out of balance, then they are all off. The mystery of PCOS is if the cysts cause hormonal imbalance or if the hormonal imbalance causes the growth of cysts. [5]

As mentioned, in Chinese medicine the three main organs connected to PCOS and infertility are Kidney, Spleen and Liver. Except the deficiency in these organs, there may be also a component of Phlegm and Dampness accumulation as seen in the physical cysts. If there is a stagnation of Blood and Qi, they can cause painful blood clots during menstruation. The treatment for every patient should be individual. To achieve the results it is needed treatments to be done for 3-6 months. [5] In our case, we succeeded to get the positive results with only 6 treatments in a period of 2 months (09.08-05.10.18). The acupuncture points used in the treatments are chosen with aim to remove the blockages that cause stagnation, improve the energy flow to the reproductive system, remove the phlegm and stasis, strengthen the immune system, strengthen the Spleen, tonify the Blood, harmonize the Liver Qi, nourish the Kidney Yin to support estrogen and improve general well-being. [6] [7] 
Acupuncture can be amazing treatment for restoring the hormonal balance, shrink ovarian cysts, increase the blood flow to the uterus and ovaries, improve the microcirculation, decrease the excessive weight and benefit ovulation and egg implantation. [5] [8]

\section{Conclusion}

Acupuncture as a treatment for infertility is very effective and gives excellent results by improving the hormonal levels, the menstruation and the ovulation in women, thus giving a chance to the patients to become pregnant spontaneously.

\section{References}

[1] Wilson RD; Can Acupuncture Treat Infertility? ; 2017 [www.healthline.com]

[2] Zhu J, Arsovska B, Sterjovska-Aleksovska A, Kozovska K; Acupuncture Treatment of Subfertility and Ovarian Endometrioma; Open Access Maced J Med Sci. 2018 Mar 15; 6(3): 519-522.

[3] Ndefo AU, Eaton A, Green RM; Polycystic Ovary Syndrome, A Review of Treatment Options with a Focus on Pharmacological Approaches; P T. 2013 Jun; 38(6): 336-338, 348, 355.

[4] Pyne A; Anna's News: A TCM View of Polycystic Ovarian Syndrome; 2010 [www.pullingdownthemoon.com]

[5] Thompson S; Treating Polycystic Ovarian Syndrome with TCM and Diet; 2013 [www.sacredvesselacupuncture.com]

[6] Zhu J, Arsovska B, Kozovska K; Acupuncture Treatment for Fertility; Open Access Maced J Med Sci. 2018 Sep 25; 6(9): 1685-1687.

[7] Zhao QL; TCM Treatment of Female Infertility Caused by High FSH; Journal of the Association of Traditional Chinese Medicine (UK); Vol 16 Issue No 1, March 2009

[8] Zhu J, Arsovska B, Kozovska K; Acupuncture treatment in menstrual disorder after oocyte donation; International Journal of Scientific Reports 2018 Aug;4(8):208-209

*Corresponding author.

E-mail address: tongdatang-tcm@ hotmail.com 